Gefässchirurgie 2009 · 14:167-168 DOI 10.1007/s00772-009-0685-1

Online publiziert: 02. Mai 2009

(c) Springer Medizin Verlag 2009

G. Rümenapf ${ }^{1,3} \cdot$ S. Morbach ${ }^{2} \cdot$ E. Müller $^{2} \cdot$ H. Reike ${ }^{2} \cdot$ A. Risse ${ }^{2} \cdot$ M. Spraul ${ }^{2}$

${ }^{1}$ Deutsche Gesellschaft für Gefäßchirurgie, Berlin

2 Deutsche Diabetes Gesellschaft, Bochum

${ }^{3}$ Gefäßzentrum Oberrhein, Diakonissen-Stiftungs-Krankenhaus, Speyer

\title{
Der diabetische Fuß - eine interdisziplinäre Aufgabe
}

ämischen Füße ist, so wichtig ist es aber auch, dass sie in ein interdisziplinäres und intersektorales Behandlungskonzept mit einvernehmlich vereinbarten Regelungen eingegliedert wird. Fehlt dieses, bleiben die langfristigen Ergebnisse aufgrund von Ulkusrezidiven und Folgeamputationen frustrierend [1]. Offensichtlich besteht trotz zunehmenden Engagements unterschiedlicher Fachgesellschaften nicht nur in Deutschland eine Koordinationsstörung bei der Behandlung des DFS [8]. Europaweit ist ein Drittel aller Patienten mit DFS zum Zeitpunkt der erstmaligen Vorstellung in einer spezialisierten Einrichtung bereits länger als 3 Monate in Behandlung gewesen. Bei Patienten mit DFS und kritischer Extremitätenischämie erfolgt nur in $56 \%$ der Fälle eine adäquate Gefäßdiagnostik und nur bei $43 \%$ eine Revaskularisation [8].

\section{- Durch bessere Koordination der Behandlung in interdisziplinären Zentren könnte die Zahl der Amputationen um mehr als $80 \%$ gesenkt werden [5].}

Eine Möglichkeit, die Aktivitäten der verschiedenen Fachgesellschaften bezüglich des DFS zu koordinieren und damit die Behandlungsergebnisse zu verbessern, ist die gemeinsame Formulierung von hochwertigen evidenzbasierten Leitlinien (EbL) [10]. Experten sichten verfügbare Studien und bewerten sie nach wissenschaftlichen Kriterien hinsichtlich ihrer Bedeutung für Klinik und Praxis. Diese Erkenntnisse gehen über den Erfahrungsschatz des einzelnen Arztes hinaus. Sie ergänzen als Entscheidungshilfe das solide medizinische Grundwissen und unterstützen die "good clinical practice“. Medizinische Abläufe sollen bei vernünftigem Ressourcenverbrauch so unterstützt werden, dass bei möglichst vielen Patienten optimale Behandlungserfolge erzielt werden.

Es gibt in Deutschland eine Leitlinienflut, häufig ohne dass sich die Fachgesellschaften bei der Formulierung abgestimmt hätten. Dies kann zu „Fächeregoismen" führen.

\section{- Nur ca. 50 Leitlinien der \\ Arbeitsgemeinschaft der wissenschaftlichen medizinischen Fachgesellschaften (AWMF) sind streng evidenzbasiert (S3).}

17 dieser Leitlinien stammen von der Deutschen Diabetes-Gesellschaft (DDG), die in dieser Hinsicht führend ist [10]. Im Rahmen dieses Leitlinienprogramms entstand im Jahre 2004 die EbL „Diagnostik, Therapie, Verlaufskontrolle und Prävention des diabetischen Fußsyndroms " [7]. Schon hier wurde die Bedeutung der gefäßmedizinischen Diagnostik und Therapie beim neuroischämischen DFS ausdrücklich betont. Da sich in der Gefäßmedizin in kurzer Zeit sehr viel verändert hat, wurde die Kommission Diabetischer Fuß der Deutschen Gesellschaft für Gefäßchirurgie (DGG) bei der Neuformulierung dieser Leitlinie beteiligt. Die neueste Fassung dieser EbL ist über die Homepage der DDG (http://www.deutsche-diabetes-gesellschaft.de) frei verfügbar. Eine Publikation in der Zeitschrift „Diabetologie und Stoffwechsel“ steht in 2009 an. 
In diesem Zusammenhang soll auch auf die im Jahr 2006 erstmals veröffentlichte Nationale Versorgungsleitlinie (NVL) „Typ-2-Diabetes - Präventions- und Behandlungsstrategien für Fußkomplikationen" hingewiesen werden [2], an deren Erstellung die Kommission Diabetischer Fuß der DGG ebenfalls beteiligt war. Die NVL zielt auf die Entwicklung und Implementierung versorgungsbereichsübergreifender Leitlinien $\mathrm{zu}$ ausgesuchten, weit verbreiteten und im Rahmen von Disease-Management-Programmen betreuten Erkrankungen ab. Dabei werden auch organisatorische Rahmenbedingungen der Patientenversorgung und das Funktionieren der Nahtstellen zwischen den verschiedenen Disziplinen und Versorgungsbereichen adressiert. Die NVL sind ein gemeinsames Projekt der Bundesärztekammer, der AWMF sowie der Kassenärztlichen Bundesvereinigung (KBV) und werden vom Ärztlichen Zentrum für Qualität in der Medizin (ÄZQ) koordiniert und entwickelt. Die Wunschkriterien zur Implementierung von Leitlinien wurden bei der obigen NVL [2] zum Großteil realisiert. Gerade die NVL hat gezeigt, dass eine partnerschaftliche $\mathrm{Zu}$ sammenarbeit von Diabetologen, Gefäßchirurgen, Angiologen, Radiologien, Allgemeinchirurgen, Orthopäden, orthopädischen Schuhmachern, Podologen, Infektiologen und Patientenvertretern erfolgreich ist.

Es sei noch erwähnt, dass die neue $\mathrm{S}_{3}$ Leitlinie PAVK der Deutschen Gesellschaft für Angiologie in Kürze publiziert wird. Auch bei der Erstellung dieser Leitlinie hat die DGG neben anderen Fachgesellschaften mitgearbeitet und damit zu einer reibungsloseren Kooperation der verschiedenen Fachgesellschaften beigetragen.

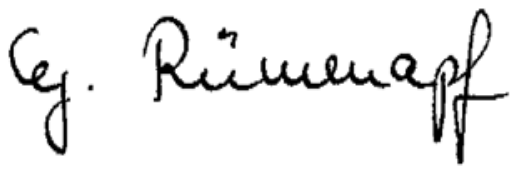

Prof. Dr. G. Rümenapf

\section{Korrespondenzadresse}

Dr. G. Rümenapf

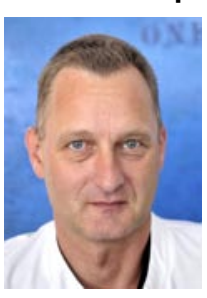

Gefäßzentrum Oberrhein, Diakonissen-StiftungsKrankenhaus Hilgardstr. 26, 57343 Speyer gerhard.ruemenapf@ diakonissen.de

\section{Literatur}

1. Apelquist J, Larsson J, Agardh CD (1993) Longterm prognosis for diabetic patients with foot ulcers. J Int Med Res 233:485-491

2. Bauer $\mathrm{H}$ et al (o J) Nationale VersorgungsLeitlinie Typ II-Diabetes - Präventions- und Behandlungsstrategien für Fußkomplikationen. http://www.diabetes.versorgungsleitlinie.de

3. Heikkinen M, Salenius JP, Auvinen O (2000) Projected workload for a vascular service in 2020. Eur JVasc Endovasc Surg 19:351-355

4. Heller G, Günster C, Swart E (2005) Über die Häufigkeit von Amputationen unterer Extremitäten in Deutschland. Dtsch Med Wochenschr 130:16881690

5. Holstein P, Ellitsgaard N, Olsen BB, Ellitsgaard V (2000) Decreasing incidence of major amputations in people with diabetes. Diabetologia 43:844-847

6. International Diabetes Federation (IDF) (2007)

The diabetes atlas, 3rd edn. Available from: http:// www.eatlas.idf.org/

7. Morbach S, Müller E, Reike H et al (2004) Diagnostik, Therapie, Verlaufskontrolle und Prävention des diabetischen Fußsyndroms. Diabetes Stoffwechsel 13:9-30

8. Prompers L, Huijberts M, Apelquist J et al (2008) Delivery of care to diabetic patients with foot ulcers in daily practice: results of the Eurodiale Study, a prospective cohort study. Diabet Med 25:700707

9. Rathmann W, Haastert B, Icks A et al (2003) High prevalence of undiagnosed diabetes mellitus in Southern Germany: target populations for efficient screening. The KORA survey 2000. Diabetologia 46:182-189

10. Scherbaum WA, Landgraf R, Selbmann HK et al (2008) Diabetes-Leitlinien. Gemeinsames Handeln aller Beteiligten. Dtsch Ärztebl 105:B1495-B1497

11. Stiegler H, Standl E, Frank S, Mendler G (1998) Failure of reducing lower extremity amputations in diabetic patients: results of two subsequent population based surveys 1990 and 1995 in Germany. VASA 27:10-14

\section{Diabetisches Fußsyndrom}

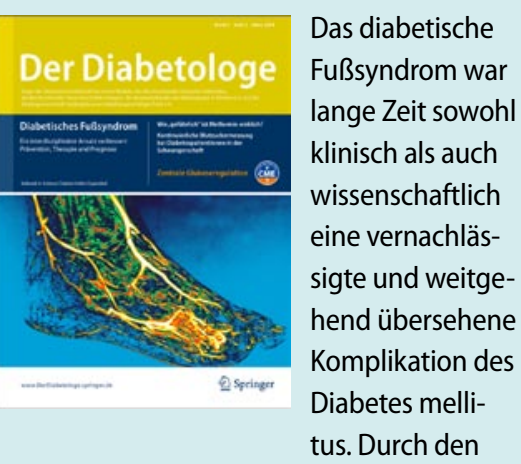

interdisziplinären Therapieansatz, der vom hausärztlichen Bereich bis hin zur spezialisierten Fachabteilung reicht, ist es möglich geworden, die Prävention, die Therapie und die Prognose des diabetischen Fußes drastisch zu verbessern.

In der Ausgabe 2/2009 der Zeitschrift „Der Diabetologe" wird die interdisziplinäre Zusammenarbeit beim diabetische Fußsyndrom umfassende dargestellt.

Das Heft enthält Beiträge u. a. zu diesen

Themenschwerpunkten:

- Gefäßchirurgische Therapie des diabetischen Fußsyndroms

- Innere Amputationen beim diabetischen Fußsyndrom

- Die Fußbehandlungseinrichtung der Deutschen Diabetes-Gesellschaft

Bestellen Sie diese Ausgabe zum Preis von EUR 32 zzgl. Versandkosten unter folgender Adresse:

Springer Customer Service Center Kundenservice Zeitschriften

Haberstr. 7

\section{Heidelberg}

Tel.: +49 6221-345-4303

Fax.: +49 6221-345-4229

E-Mail: leserservice@springer.com 